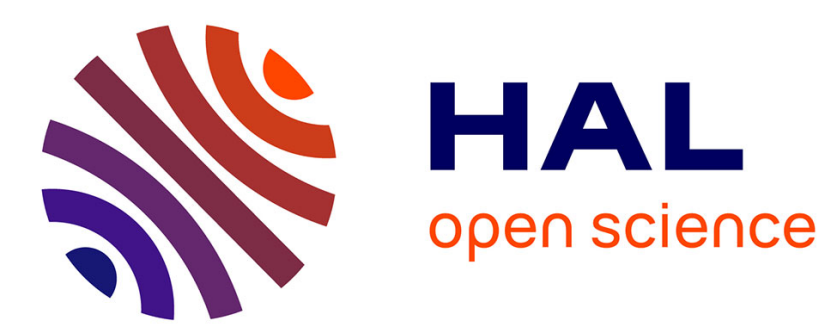

\title{
LOGEMENT SOCIAL NIPPON : UN BILAN APRÈS LA CATASTROPHE DU 11 MARS 2011
}

\author{
Cécile Asanuma-Brice
}

\section{To cite this version:}

Cécile Asanuma-Brice. LOGEMENT SOCIAL NIPPON : UN BILAN APRÈS LA CATASTROPHE DU 11 MARS 2011. Le Japon: de nouveaux choix sociaux? Les enjeux des politiques sociales, 2011. hal-02611134

\section{HAL Id: hal-02611134 https://hal.science/hal-02611134}

Submitted on 18 May 2020

HAL is a multi-disciplinary open access archive for the deposit and dissemination of scientific research documents, whether they are published or not. The documents may come from teaching and research institutions in France or abroad, or from public or private research centers.
L'archive ouverte pluridisciplinaire HAL, est destinée au dépôt et à la diffusion de documents scientifiques de niveau recherche, publiés ou non, émanant des établissements d'enseignement et de recherche français ou étrangers, des laboratoires publics ou privés. 


\section{LOGEMENT SOCIAL NIPPON : UN BILAN APRÈS LA CATASTROPHE DU 11 MARS 2011}

Cécile Asanuma-Brice

Caisse nationale d'allocations familiales (CNAF) | «Informations sociales »

2011/6 n 168 | pages 34 à 44

ISSN 0046-9459

Article disponible en ligne à l'adresse :

https://www.cairn.info/revue-informations-sociales-2011-6-page-34.htm

\section{Pour citer cet article :}

Cécile Asanuma-Brice, « Logement social nippon : un bilan après la catastrophe du 11 mars 2011 », Informations sociales 2011/6 ( $\mathrm{n}^{\circ}$ 168), p. 34-44.

Distribution électronique Cairn.info pour Caisse nationale d'allocations familiales (CNAF).

(C) Caisse nationale d'allocations familiales (CNAF). Tous droits réservés pour tous pays.

La reproduction ou représentation de cet article, notamment par photocopie, n'est autorisée que dans les limites des conditions générales d'utilisation du site ou, le cas échéant, des conditions générales de la licence souscrite par votre établissement. Toute autre reproduction ou représentation, en tout ou partie, sous quelque forme et de quelque manière que ce soit, est interdite sauf accord préalable et écrit de l'éditeur, en dehors des cas prévus par la législation en vigueur en France. Il est précisé que son stockage dans une base de données est également interdit. 


\section{Logement social nippon : un bilan après la catastrophe du 11 mars 2011}

Cécile Asanuma-Brice - géographe urbaine

En 1923, le grand tremblement de terre du Kantô avait donné une impulsion définitive en faveur de la mise en place d'un système de financement public du logement. Cet organisme, qui s'éloignait depuis les années 1990 de sa mission sociale pour privilégier la rentabilité de son parc, a été brutalement rappelé à sa vocation par les catastrophes du 11 mars 2011. Dans un environnement chaotique, le gouvernement est contraint, aujourd'hui, de rénover le parc de logements sociaux vacants qu'il vouait à démolition.

Les catastrophes naturelles et humaines ont été à l'origine de la mise en place d'un système de logements publics au Japon, il y a maintenant presque un siècle. Ces derniers temps, cette organisation sociale semblait s'essouffler aux yeux des autorités qui tendaient à opter pour une gestion américanisée du social, se désinvestissant peu à peu tout en promouvant l'action de nouveaux acteurs privés, comme celle des associations à but non lucratif. On peut se demander si le grand tremblement de terre survenu le 11 mars 2011 dans l'est du Japon va remettre en cause ce désengagement annoncé de l'État dans le secteur social, et plus spécifiquement dans le logement social. L'analyse du rôle des organismes publics de logement et des politiques mises en œuvre pour gérer cette nouvelle crise est essentielle pour comprendre la remise en cause d'un système en pleine mutation. La crise actuelle pourrait être l'occasion de donner un nouveau souffle à une organisation prématurément condamnée.

Naissance et croissance du logement collectif public

La naissance des premiers logements publics japonais est due au grand tremblement de terre du Kantô de 1923. L'insuffisance du nombre de logements, auparavant déjà très lourde, devient alors criante. Les solutions doivent être rapides pour faire face au nombre grandissant de sans-abri. En 1924, le gou- 
vernement crée l'Association pour l'enrichissement du peuple, dont le rôle est d'inciter à la construction de logements pour les victimes du tremblement de terre.

Malgré le faible nombre de logements construits par cette association (12 000 logements en accession à la propriété ou en locatif en dix-huit ans), elle fut précurseuse à plus d'un titre. D'abord parce qu'il s'agit du premier organisme de production de logements publics à proprement parler; ensuite en raison des améliorations architecturales qu'elle a apportées (petits collectifs ignifuges en béton armé); et enfin pour les innovations urbanistiques et sociales mises en œuvre, chaque opération ayant été traitée comme un quartier autonome possédant ses propres commerces et équipements collectifs à l'intérieur même des immeubles d'habitation. Les appartements sont conçus pour s'adapter à tous types de famille, des célibataires aux couples avec enfants (Bourdier, 1992). Bien que situés essentiellement en centre-ville, ils furent les premiers logements collectifs publics intégrant les notions de modernisation et de fonctionnalisation des espaces, apparues dans les années 1920. La Seconde Guerre mondiale représente la seconde impulsion décisive donnée à la politique de production de logements. Il devient de nouveau nécessaire de répondre à une situation dramatique. C'est dans ce contexte qu'apparaît l'habitat public de gestion municipale, toujours en vigueur, avec en 1951 la promulgation de la loi sur les logements municipaux. Cette loi spécifie l'approvisionnement de logements de gestion municipale destinés à la classe sociale ayant les revenus les plus faibles. Il est à noter que c'est la première fois qu'une loi sur le logement prend comme critère premier le bas salaire des habitants. Jusque-là, la logique motrice de la production publique de logements n'était liée qu'au seul manque de logements et non pas au montant des loyers.

Puis, dans une logique de plans quinquennaux, est créé l'Organisme public du logement et des équipements urbains. Il dépend directement du gouvernement et a pour fonction de produire des logements à proximité de Tokyo pour les cols bleus venant travailler dans la capitale. Dans les faits, cet outil direct du gouvernement produit le prototype d'habitat moderne décrit par les directives édictées par l'État. Ces habitats modèles sont ensuite reproduits par les organismes publics municipaux et départementaux dans la mesure de leur capacité budgétaire, mais également par les constructeurs de logements collectifs privés.

Les danchi, ou grands ensembles, naissent de ces deux organismes. Dans la forme, ils répondent au désir de modernité du peuple, avec l'apparition de la dining kitchen (cuisine à l'occidentale), l'affirmation de la culture de la chaise ou encore des salles de bain individuelles (remplaçant le sentô, bain public très répandu au Japon), etc.

Le confort occidental prend place au sein du foyer et, avec lui, une volonté de la part des gestionnaires publics de vouloir obtenir toujours plus de 
rentabilité économique, à l'heure où le Japon connait une croissance explosive. La forme des bâtiments sera finalement dictée par ces logiques du gain dans les années 1980-1990. Elle donne naissance à des cités de logements toujours plus denses, des espaces communs dénués de fonctions, en nombre réduit et privés de toute convivialité. Les conséquences de ces formes urbaines sont sensibles, notamment dans la modification des rapports de voisinage, qui se distendent à partir d'un certain seuil de densité trop forte, comme répondant à une régularisation naturelle. Le stress né de ces espaces oppressants, les pertes de lien avec le lieu d'implantation des logements, l'appropriation impossible d'espaces anonymes trop vastes ou encore le sentiment d'insécurité sont autant de problématiques que nous connaissons bien dans nos cités de logements publics français.

Néanmoins, les sociétés de construction de ces logements continuent plus que jamais à jouer sur des images. Des cités où seul le béton fleurit portent le nom de « Green Town», «Belle vallée », « Promenade »...

\section{Du logement public aux logiques privées}

Le Japon compte aujourd'hui 3119000 logements publics, contre 4300000 en France. Comme nous l'avons décrit au préalable, l'organisation du logement public japonais a été instituée dès les années 1950. On peut la diviser en deux grands groupes: les kôei jûtaku, ou logements publics municipaux et départementaux, et les logements relevant de l'Organisme public du logement et des équipements urbains.

\section{Les kôei jûtaku: une logique sociale imposée}

Au nombre de 2183000 logements pour l'ensemble du territoire, ceux relevant des collectivités locales représentent $68 \%$ du parc de logements publics et $12 \%$ du parc locatif total. Les personnes postulantes doivent avoir un salaire inférieur à 150000 yens par mois (soit 1166 euros) et leur sélection se fait par tirage au sort.

On note que le plafond retenu est relativement bas en comparaison des normes françaises (2 025 euros par mois pour une personne vivant seule à Paris et dans les communes limitrophes et 1761 euros pour une personne seule en province, selon un processus d'évolution dégressive en fonction du nombre de personnes à charge).

Ces logements ont donc véritablement une vocation sociale si l'on considère le revenu moyen des Japonais qui, selon le ministère du Travail, est de 437000 yens par mois (3 289 euros), avec une différence notable de $538000 \mathrm{JPY}$ pour les hommes (4 050 euros) et $273000 \mathrm{JPY}$ pour les femmes (2 055 euros).

La vocation sociale que nous évoquions a été préservée pour deux raisons. L'une répond, dans certains cas, à une réelle volonté politique de soutien social. C'est ainsi le cas du département de Kanagawa, et notamment de la 
ville de Yokohama, qui a fondé son image sur l'assistance sociale apportée à sa population. Ce département au sud de Tokyo et les communes qui le constituent proposent 102000 logements publics en locatif pour une population de 8,9 millions d'habitants, soit 7,5\% du nombre total de logements locatifs, à

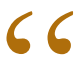
peine en dessous du taux proposé par la ville de Tokyo, qui est de $10 \%$ du logement locatif total (277 000 logements dépendants de la capitale pour 12839000 habitants). L'autre raison, moins philanthropique, dépend de l'incapacité budgétaire du département ou des communes gestionnaires à renouveler leur parc de logements publics. Ils ont été contraints de maintenir des loyers particulièrement bas, sous peine de devoir gérer la vacance de trop nombreux appartements vétustes. On peut observer cet état de fait plus particulièrement dans les départements de Saitama, au nord de Tokyo, qui compte 37000 logements dépendants de la commune ou du département, et de Chiba, péninsule à l'est de Tokyo, avec 34000 logements dépendants de la commune ou du département. De façon générale, les communes aujourd'hui ne s'aventurent pas dans la construction de logements publics neufs.

\section{Les logements de l'Organisme public d'équipement et de logement}

Cet organisme a changé de nom à plusieurs reprises pour devenir enfin, en 2005, l'Organisme de renouvellement urbain (Toshi saisei Kikố). En même temps qu'il s'orientait vers une privatisation lente mais certaine, il s'est vu destitué en grande partie de sa charge logements pour ne garder que le rôle d'aménageur urbain. S’il a cessé l'achat de nouveaux équipements et terrains dès 2001, il garde néanmoins le pouvoir de rénover les lotissements qu'il possède déjà et n'a pas hésité à le faire.

Avec 768608 logements en sa possession, cet organisme reste l'un des plus grands propriétaires mondiaux. Comptant 4000 employés, il se caractérise par sa centralisation, laquelle engendre des lourdeurs incontestables dans son fonctionnement.

Répondant à des logiques d'aménagement urbain national (la répartition de ses cités de logements en témoigne : elles comptent environ 85000 logements dans les trois départements autour de Tokyo et 220000 dans la capitale), cet organisme s'est peu à peu éloigné des logiques locales et ses orientations ont finalement privilégié des volontés de rentabilité foncière. Les années 1990-2000 marquent ainsi un changement de politique important. Progressivement privé de sa prérogative sociale, il se consacre à une politique d'investissement et de rénovation du parc de logements construits en 1955. Multipliant par trois ou quatre les loyers d'origine, cette décennie fut particulièrement impopulaire. À titre d'exemple, le loyer d'un logement de $70 \mathrm{~m}^{2}$ dans une immense tour au sud de Tokyo 
(Nakameguro) montait jusqu'à 250000 yens (1 910 euros) par mois. Si la sélection des personnes postulantes s'effectue là aussi par tirage au sort, ce qui peut être considéré comme une méthode égalitaire, il leur est en revanche demandé de justifier de garanties équivalant au minimum à quatre fois le montant du loyer mensuel. Il n'était donc plus question de social et c'était à une nouvelle clientèle, plus solvable, à qui feu Urban Renewal (Toshi Seibi Kôdan, nom du principal organisme d'État de rénovation urbaine et de production/gestion de logements) proposait désormais ses services.

Mais avec l'essoufflement de la période de prospérité économique et l'augmentation régulière du nombre de logements vacants, une telle politique était devenue par trop risquée. L'État décide de se désengager après la publication d'un bilan désastreux. Les rénovations sont alors limitées à 3500 par an et l'organisme se réoriente vers une nouvelle politique de réhabilitation de l'ancien parc de logements vacants. Dans ce domaine, il reste précurseur, notamment en matière de kôei jûtaku, car il dispose de moyens budgétaires que les collectivités locales n'ont pas.

Les événements du 11 mars 2011 vont néanmoins renverser une nouvelle fois la situation. Les logements vacants tant décriés vont permettre le relogement d'une partie des 126000 réfugiés et remettre à l'ordre du jour la nécessité d'une politique publique du logement.

\section{Conséquences du grand tremblement de terre} dans l'est du Japon

Selon une estimation du ministère du Territoire, des Infrastructures et des Transports datée du 15 mai 2011(1), le nombre de personnes décédées serait de 15 057, celui de personnes disparues de 9121 , et celui des blessés avoisinerait les 5282 personnes. Deux mois après les événements, 126372 personnes se sont réfugiées soit dans des structures publiques réquisitionnées ou simplement abandonnées (gymnases d'écoles, musées, etc.), soit dans des abris précaires. Un certain nombre d'entre elles occupent notamment une tente au premier étage de leur ancienne demeure ravagée qu'elles ne souhaitent pas quitter.

\section{Bilan des dégâts dans le secteur du logement}

Le nombre de logements détruits, le plus souvent emportés par le tsunami ou brûlés par les incendies multiples engendrés par la triple catastrophe du 11 mars 2011, avoisinerait les 90000 unités, selon les 8471 enquêteurs qui ont parcouru les dix départements concernés, soit 149 villes et villages. Les logements " endommagés de moitié », selon les termes du ministère, seraient au nombre de 36 104. Il faut ajouter à ce bilan 8951 habitations endommagées par les inondations. Néanmoins, ce bilan des plus alarmants ne couvre pas l'ensemble des zones sinistrées, les 
données n'étant pas encore disponibles pour l'ensemble des communes des départements d'Iwate et de Miyagi, les plus touchées par le tsunami. Déterminer l'ampleur du désastre est une tâche difficile car les propriétaires des bâtiments sont, pour certains, décédés, portés disparus ou réfugiés dans des lieux inconnus, tandis que les locaux administratifs contenant les plans du cadastre (préfectures, mairies, etc.) ont été, pour la plupart, emportés par les flots.

\section{Répartition géographique des dégâts}

Concernant la répartition géographique des destructions et des besoins qui en résultent, la zone principalement touchée est celle du Tohoku, une région faisant face à l'épicentre du tremblement de terre du 11 mars, cible du tsunami. On y compte 87835 logements totalement détruits ${ }^{(2)}$ et 24290 logements à moitié détériorés (les flots du tsunami n'ont parfois atteint qu'un ou deux étages des résidences, emportant tout sur leur passage mais épargnant les autres étages).

Les préfectures les plus touchées sont évidemment celles qui ont subi, coup sur coup, au moins deux des trois catastrophes (tremblement de terre, tsunami et explosion de la centrale nucléaire Fukushima Dai-ichi). C'est le cas principalement de Miyagi, qui compte 61797 logements détruits, 14333 habitats endommagés et 47383 personnes réfugiées ${ }^{(3)}$, suivi par les départements d'Iwate (17 127 logements détruits, 2636 habitations à moitié endommagées et 44821 réfugiés) et de Fukushima, où l'on a recensé un nombre comparativement faible d'habitats détruits en totalité ou en partie (respectivement 8601 et 6 254), mais où le nombre de réfugiés reste important en raison des déplacements de

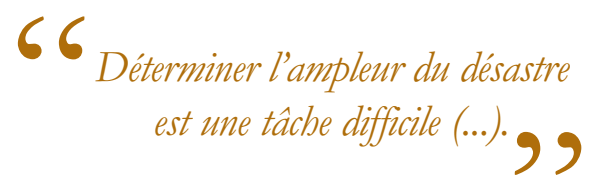
population engendrés par la catastrophe nucléaire, qui concernaient 24809 habitants en juin 2011. Bien que les mouvements de population ne soient pas encore stabilisés dans cette région, on peut estimer que, pour l'ensemble de ces trois départements, au minimum 120000 personnes devront être relogées.

Par ailleurs, les enquêtes menées par le ministère du Territoire, des Infrastructures et des Transports se poursuivent pour recenser le nombre d'habitations détériorées par les nombreux séismes et répliques. Une gradation sur trois niveaux a été établie afin de déterminer le degré d'habitabilité des logements.

Le graphique ci-après répertorie en marron les habitats inspectés mais ne présentant pas de défauts notables, en beige les logements nécessitant des travaux et en gris ceux estimés comme dangereux, dans lesquels il est interdit de pénétrer. Au total sont actuellement décomptés 1382 logements classés gris, 2159 beiges et 2441 marron. Miyagi accuse le bilan le plus lourd avec 836 unités classées gris, 1451 beiges et 1522 marron. 


\section{Évaluation de l'état des logements non détruits dans la zone sinistrée}

(échantillon de 5982 logements)

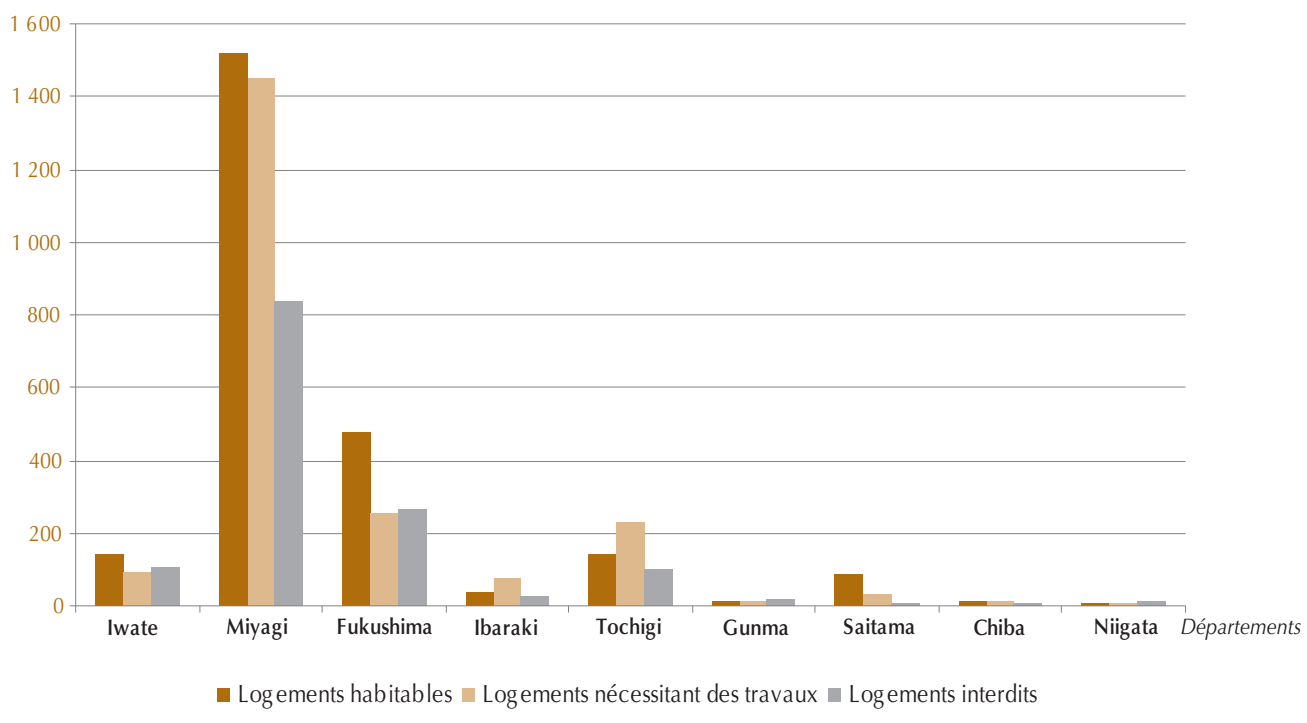

De nombreuses habitations à moitié endommagées sont également à déplorer dans les départements d'Ibaraki et de Chiba, notamment en raison du phénomène de liquéfaction des sols qui a principalement touché les terrains gagnés sur la mer à l'intérieur de la baie de Tokyo (autour de la commune d'Urayasu). Les vibrations du sol engendrées par le séisme entrainnent une désolidarisation des grains de sable, permettant la remontée de l'eau à la surface des terrains. L'eau jaillit de toute part, fissurant les matériaux de surface, quelle qu'en soit la nature. Le sol devenu désormais très instable ne peut plus assurer son rôle de dalle. Routes et trottoirs se cassent et les bâtiments ou tout autre élément posé au sol s'enfoncent lentement dans une matière boueuse mouvante.

À la suite des nombreuses secousses sismiques qui ont perduré pendant plus de deux mois après le grand tremblement de terre, hormis les constructions totalement détruites et celles qui le sont à moitié, beaucoup d'autres ont aussi subi des dommages non négligeables et nécessitent d'importantes réparations avant de pouvoir être de nouveau habitées. Cela explique l'affluence des personnes réfugiées dans les départements de Gunma (2 797 personnes), de Saitama (3 458 personnes) et de Niigata (4 919 personnes). Ce dernier a en outre été victime d'un tremblement de terre (il ne s'agissait pas d'une réplique mais bien d'un autre séisme) de magnitude 6 à 7 peu après celui du 11 mars, engendrant à son tour de nombreux dégâts.

Bilan des dégâts dans le secteur du logement public

Parmi les logements détruits, le ministère du Territoire, des Infrastructures et 
des Transports a recensé, dans les 12 départements touchés, 403 bâtiments communaux ou publics (kôer) totalement détruits, soit 1290 logements, et 2155 constructions détruites à moitié ou partiellement, ce qui représente 21264 unités de logement. Aucun logement appartenant au groupe UR (Urban Renewal) n'aurait été détruit totalement, mais 30547 habitations de ce groupe auraient néanmoins été endommagées, dont un grand nombre dans la ville de Tokyo (15 725).

\section{Vers la reconstruction : le rôle des organismes publics}

Dans le cas d'une catastrophe nationale telle que celle du 11 mars, l'organisme chargé de centraliser les informations et de coordonner la politique en termes d'habitat est le ministère du Territoire, des Infrastructures et des Transports. La difficulté de la tâche qui lui est allouée réside dans l'évaluation du nombre de logements nécessaires à la construction. En effet, l'ampleur des dégâts ne permet toujours pas de déterminer avec exactitude le nombre de personnes décédées et leur identité. Ainsi, le nombre de demeures détruites n'est pas en relation directe avec celui de logements qu'il est nécessaire de construire. Quant au nombre de personnes réfugiées, celui donné reste une approximation qui ne prend en compte que les personnes que l'on a pu identifier comme telles. Enfin, les mouvements de population continuent en raison de la contamination nucléaire qui a touché le département de Fukushima et qui continue de se répandre. La zone d'évacuation de 20 à $30 \mathrm{~km}$ autour de la centrale ne correspond pas aux dépôts réels d'éléments radioactifs, dont on connaît maintenant la répartition avec précision. Ainsi, les personnes informées qui peuvent faire face au coût d'un déménagement ainsi qu’à la perte de leur emploi cherchent à se reloger dans des lieux où le risque sanitaire est moins grand.

À l'aide d'échelles numériques, qui ne restent que des évaluations à un instant $\mathrm{T}$ permettant de donner un ordre de grandeur, les autorités publiques ont mis en place deux volets d'action concernant le relogement des victimes : la mise à disposition gratuite de logements publics vacants et la construction d'habitats d'urgence.

\section{La mise à disposition des logements vacants}

À l'échelle nationale, chaque ville a fait part au département dont elle dépend du nombre d'habitats municipaux vacants dont elle dispose. Les départements ont fait de même avec les logements vacants relevant de leur gestion, dont ils ont déclaré le nombre total au ministère du Territoire. Pour l'ensemble du pays, 22000 logements vacants relevant des communes ou des départements ont ainsi été mis à disposition gratuitement pour une période variant de six mois à un an. Mille sept cents de ces logements se trouvent dans le département du Tohoku. L'organisme Urban Renewal (UR) a, quant à lui, offert 5100 logements vacants gratuits pour des durées similaires, 
dont 130 se trouvent dans le Tohoku. L'attribution de ces logements se fait par tirage au sort. Deux mois après le séisme, seuls 4800 logements municipaux et départementaux et 730 logements de la UR ont été attribués(4). Le nombre de foyers effectivement relogés dans ces logements est donc restreint.

De multiples causes sont à l'origine de la lenteur du relogement des personnes réfugiées. La première relève de la difficulté à faire circuler l'information dans les divers lieux de refuge, lesquels ne sont pas tous identifiés. La grande majorité ne sont pas reliés au réseau Internet, vecteur essentiel de l'information, et n'ont pas connaissance de ces possibilités de relogement. Par ailleurs, la participation au tirage au sort nécessite de pouvoir se déplacer, à plusieurs reprises en cas d'échec, parfois relativement loin, ce que ces personnes ne sont pas toujours en mesure de faire. Enfin, ces habitants souhaiteraient, pour la plupart, pouvoir être relogés à proximité de leur ancien lieu d'habitation.

Si les bâtiments ont été détruits, les réseaux communautaires, eux, sont encore bien établis et occupent une fonction de repère social parfois vitale. Or, un grand nombre des logements vacants proposés se trouvent très éloignés des lieux touchés par le séisme. Les agglomérations d’Osaka (2 844), Hokkaidô (2 154), et enfin Tokyo (1 120 UR) sont les plus offrantes. Un tel éloignement du lieu premier d'habitation signifie une reconstruction totale de l'environnement social des personnes déplacées. Si cela semble envisageable pour des personnes jeunes, la difficulté d'une réinsertion complète dans un nouvel environnement est quasi insurmontable pour des personnes âgées. Cela a été démontré à la suite du séisme de Kobe et les lourdes conséquences de relogements inadéquats restent encore visibles aujourd'hui, entraînant suicides et alcoolisme au sein de ces populations peu mobiles. Or, l'âge moyen de la population des campagnes du nord de Honshu touchées par les séismes, le tsunami et l'explosion de la centrale est assez élevé. Selon le dernier recensement national de la population, sur 47 départements classés par ordre d'âge croissant, le département d'Iwate occupe la $40^{\circ}$ place, avec un âge moyen de 45,5 ans, alors que la population de Fukushima a un âge moyen de 44,2 ans et celle de Miyagi de 43 ans. Il est donc difficile d'envisager des déplacements lointains.

Enfin, l'aspect provisoire de la gratuité semble également être un frein. Les populations s'interrogent sur l'après et la façon dont elles pourront financer leur loyer lorsque la période de gratuité aura pris fin.

\section{Les logements provisoires}

De même que pour la disponibilité en logement public, chaque commune dépose sa demande de logements provisoires au département qui centralise à son échelle avant de renvoyer l'information au ministère du Travail (5). Ces logements sont financés par la Caisse spéciale d'aide en cas de cataclysme. 
Les données sont ensuite fournies au ministère du Territoire, des Infrastructures et des Transports, qui fait appel à des promoteurs privés pour la construction. Le nombre de logements provisoires d'urgence requis est estimé, au mois de mai 2011, à 14000 logements pour le département d'Iwate, 30000 pour celui de Miyagi, 24000 pour celui de Fukushima, 20 à Tochigi, 230 à Chiba, et 55 à Nagano, soit un total de 68305 logements( ${ }^{(6)}$. Deux mois après les événements, concernant les principaux sites : 9634 logements provisoires répartis sur 181 sites ont été entrepris dans le département d'Iwate, 13025 à Miyagi sur 173 sites et 9590 sont en cours de réalisation à Fukushima dans 105 quartiers. Au total, 13783 logements étaient achevés mi-mai (3 433 à Iwate, 7445 à Miyagi, 2640 à Fukushima).

La loi fixant les règles fondamentales en matière de construction (kenchikukijunhôjo) limite la durée d'habitation dans ces logements provisoires à deux ans maximum. Si ces logements sont une solution d'urgence au relogement des populations qui en ont le plus besoin, comme les couples avec des enfants en bas âge pour qui la promiscuité des lieux de refuge est quasi insupportable (les mères témoignent notamment du stress engendré par les pleurs de leurs enfants qui réveillent les autres personnes réfugiées) et d'autres populations fragilisées, des voix mettent néanmoins en garde contre la précarité de ces abris, parfois établis sur des sites atteints par le tsunami. Par ailleurs, la rapidité avec laquelle ces logements sont construits ainsi que le manque de matériaux auquel le Japon fait face aujourd'hui (l'entreprise de laine de verre utilisée pour l'isolation des maisons a été emportée par le tsunami) sont autant d'éléments qui nous permettent d'appréhender la faible qualité de ces cellules alignées en bande sur de vastes terrains plats. L'inquiétude de voir ces habitations perdurer plus longtemps qu'elles ne le devraient est justifiée si l'on en croit les expériences passées. Il reste aujourd'hui une cinquantaine de centres d'accueil qui regroupent environ 3700 personnes.

Si l'État investit dans la construction de logements d'urgence, il ne semble pas que la décision de cesser la construction de logements publics soit remise en cause pour le moment. Les logements publics ont néanmoins une raison d'être qui se révèle quasi nécessaire quant au relogement dans les zones dévastées. Les choix et propositions de l'État en la matière seront déterminants pour les habitants de ces départements qui, par la destruction de l'ie, notion qui désigne à la fois le «foyer » et la famille qui y réside, sont confrontés à un anéantissement matériel mais également social et spirituel. 
Notes

1 - http://www.mlit.go.jp/common/000139083.pdf

2 - Recensement du ministère du Territoire, des Infrastructures et des Transports au 15 mai 2011, http://www.mlit.go.jp/common/000139083.pdf, p. 23.

3 - Recensement de la police nationale au 13 avril 2011.

4-Chiffres au 19 mai 2011.

5 - http://www.kenchiku-bosai.or.jp/jimukyoku/Oukyu/Oukyu.htm, 16 mars 2011.

6 - Voir le rapport du ministère du Territoire, des Infrastructures et des Transports, disponible sur http://www.mlit.go.jp/common/000142177.pdf

\section{Bibliographie}

- Association pour la prévention des désastres dans la construction au Japon (Nihon Kenchiku bôsai kyôkai), rapport du 16 mars 2011.

- Berque A., 2010, Histoire de l'habitat idéal. De l'Orient vers l'Occident, Paris, Le Félin, coll. "Les marches du temps».

- Bourdier M., 1992, Dôjunkai apâto no genkei - Nihon kenchiku shi ni okeru yakuwari (Le rôle des appartements de la Dôjunkai dans I'histoire de l'architecture japonaise), Tokyo, Sumai no Toshokan shuppan kyoku, août.

- Ministère du Territoire, des Infrastructures et des Transports, 2011, «Désastres dus au grand tremblement de terre de l'est du Japon » (Higashi Nihon daishinsai), rapport, version de mai 2011 ; " Réponses d'urgences aux dégâts ", rapport, section Logement, mai.

- Police nationale, « Recensement du nombre de victimes au 13 avril 2011 ». 